\title{
Chaos control in mechanical systems
}

\author{
Marcelo A. Savi ${ }^{\mathrm{a}, *}$, Francisco Heitor I. Pereira-Pinto ${ }^{\mathrm{b}}$ and Armando M. Ferreira ${ }^{\mathrm{b}}$ \\ ${ }^{a}$ Universidade Federal do Rio de Janeiro, COPPE, Department of Mechanical Engineering, 21.941.972, Rio de \\ Janeiro, RJ, P.O. Box 68.503, Brazil \\ ${ }^{\mathrm{b}}$ Department of Mechanical and Materials Engineering, Instituto Militar de Engenharia, 22.290.270, Rio de \\ Janeiro, RJ, Brazil
}

\begin{abstract}
Chaos has an intrinsically richness related to its structure and, because of that, there are benefits for a natural system of adopting chaotic regimes with their wide range of potential behaviors. Under this condition, the system may quickly react to some new situation, changing conditions and their response. Therefore, chaos and many regulatory mechanisms control the dynamics of living systems, conferring a great flexibility to the system. Inspired by nature, the idea that chaotic behavior may be controlled by small perturbations of some physical parameter is making this kind of behavior to be desirable in different applications. Mechanical systems constitute a class of system where it is possible to exploit these ideas. Chaos control usually involves two steps. In the first, unstable periodic orbits (UPOs) that are embedded in the chaotic set are identified. After that, a control technique is employed in order to stabilize a desirable orbit. This contribution employs the close-return method to identify UPOs and a semi-continuous control method, which is built up on the OGY method, to stabilize some desirable UPO. As an application to a mechanical system, a nonlinear pendulum is considered and, based on parameters obtained from an experimental setup, analyses are carried out. Signals are generated by numerical integration of the mathematical model and two different situations are treated. Firstly, it is assumed that all state variables are available. After that, the analysis is done from scalar time series and therefore, it is important to evaluate the effect of state space reconstruction. Delay coordinates method and extended state observers are employed with this aim. Results show situations where these techniques may be used to control chaos in mechanical systems.
\end{abstract}

Keywords: Chaos, control, mechanical system, nonlinear pendulum, state space reconstruction

\section{Introduction}

Nature is full of nonlinearities, responsible for a great variety of responses in natural systems. Natural rhythms could be either periodic or irregular over time and space. Each kind of dynamical behavior related to natural systems may be related with both normal and pathological physiological functioning. Extremely regular dynamics may be associated with diseases including periodic breathing, certain abnormally heart rhythms, cyclical blood diseases, epilepsy, neurological tics and tremors. On the other hand, there are phenomena where regular dynamics reflect healthy behavior as sleep-wake cycle and menstrual rhythms. Moreover, irregular rhythms can also reflect disease: cardiac arrhythmias, such as fibrillation, and different neurological disorders $[12,16]$. Chaos has an intrinsically richness related to its structure and, because of that, there are benefits for a natural system of adopting chaotic regimes with their wide range of potential behaviors. Under this condition, the system may quickly react to some new situation, changing conditions and their response [11]. Therefore, chaos and many regulatory mechanisms control the dynamics of living systems, conferring a great flexibility to the system.

Inspired by nature, the idea that chaotic behavior may be controlled by small perturbations of some physical parameter makes this kind of behavior to be desirable in different applications. Mechanical systems constitute a class of system where it is possible to exploit these ideas. Smart systems, biomechanical and robotics devices, aerospace

\footnotetext{
*Corresponding author. E-mail: savi@ufrj.br.
} 
hardware and drilling processes are some examples where chaotic behavior may appear and its control may be interesting for some specific application. Then, these systems would present a great flexibility when controlled, being able to quickly change from one kind of response to another.

Chaos control is based on the richness of responses of chaotic behavior. A chaotic attractor has a dense set of unstable periodic orbits (UPOs) and the system often visits the neighborhood of each one of them. Moreover, chaotic response has sensitive dependence to initial condition, which implies that the system's evolution may be altered by small perturbations. Therefore, chaos control may be understood as the use of tiny perturbations to stabilize an UPO embedded in a chaotic attractor, which makes this kind of behavior to be desirable in a variety of applications, since one of these UPOs can provide better performance than others in a particular situation. It should be pointed out that it is not necessary to have a mathematical model to achieve the control goal since all control parameters may be resolved from time series analysis.

Chaos control methods may be classified as discrete or continuous techniques. The first chaos control method had been proposed by Ott et al. [29], nowadays known as the OGY (Ott-Grebogi-Yorke) method. This is a discrete technique that considers small perturbations promoted in the neighborhood of the desired orbit when the trajectory crosses a specific surface, such as some Poincaré section. On the other hand, continuous methods are exemplified by the so called delayed feedback control, proposed by Pyragas [33], which states that chaotic systems can be stabilized by a feedback perturbation proportional to the difference between the present and a delayed state of the system.

There are many improvements of the OGY method that aim to overcome some of its original limitations, as for example: control of high periodic and high unstable UPO [21,28,34] and control using time delay coordinates [9, $23,38]$. For more details on chaos control based on OGY method refers to [4-8,10,17,37].

Literature presents some contributions related to the analysis of chaos control in mechanical systems. Andrievskii and Fradkov [1] present an overview of applications of chaos control in various scientific fields. Mechanical systems are included in this discussion presenting control of pendulums, beams, plates, friction, vibroformers, microcantilevers, cranes, and vessels. Moon et al. [27] analyze chaos control in an oscillator with dry friction changing the normal force with a magnetic actuator. Begley and Virgin [3] employs the OGY method to control the impact oscillator using friction force as the control parameter. $\mathrm{Hu}$ [20] discuss the control of the chaotic motion of a periodically forced mechanical system having non-smooth restoring force due to elastic stops or clearances, and the control parameter is small time-dependent perturbation. Wang and Jing [44] consider Lyapunov function method for designing a controller to control a pendulum system with chaotic motion. The desirable orbits of a pendulum are stabilized with feedforward and feedback control in references [41,42]. Spano et al. [39] exploits the ideas of chaos control applied to intelligent systems employing the OGY method to a buckled beam built with a magnetic material that changes its Young's modulus when the magnetic field is modified. Macau [26] shows that chaos control techniques can be used to efficiently keep a spacecraft around another body performing elaborate orbits.

This contribution revisits the possibility to apply chaos control principles in mechanical systems. As an application of the general idea, it is considered the analysis of chaos control in a nonlinear pendulum that is based on the experimental apparatus previously analyzed in [13,32]. This article presents a collection of results previously shown in $[30,31]$. The cited pendulum has both torsional stiffness and damping. All signals are generated numerically by the integration of the equations of the mathematical model proposed, which uses experimentally identified parameters. The close-return (CR) method [2] is employed to determine the UPO embedded in the attractor. A variation of the OGY technique called semi-continuous control (SCC) method, proposed by Hübinger et al. [21] and extended by Korte et al. [23], is considered to stabilize the desirable orbit. The control analysis considers two different situations: all state variables are available; and just a scalar time series is available. For the second situation, state space reconstruction is done with the method of delay coordinates [40] and also considering extended state observers [19]. Results confirm the possibility of the use of this approach to deal with mechanical systems.

\section{Chaos control method}

The control of chaos can be treated as a two-stage process. The first stage is composed by the identification of UPOs and is named as "learning stage". Since UPOs are system invariants, they can be analyzed from phase space reconstructed from a scalar time series [18]. 
This article considers the close-return (CR) method [2] for the detection of UPOs embedded in the attractor. The basic idea is to search for a period $-P$ UPO in the time series represented by vectors $\left\{u_{i}\right\}_{i=1}^{N}$. The identification of a period $-P$ UPO is based on a search for pairs of points in the time series that satisfy the condition $\left|u_{i}-u_{i+P}\right|_{i=1}^{(N-P)} \leqslant r_{1}$ where $r_{1}$ is the tolerance value for distinguishing return points. After this analysis, all points that belong to a period $-P$ cycle are grouped together. During the search, the vicinity of a UPO may be visited many times, and it is necessary to distinguish each orbit, remove any cycle permutation and to average them in order to improve estimations as shown in [28].

After the identification of a UPO, one can proceed to the next stage of the control process that is the stabilization of the desired orbit. The OGY approach [29] considers the stabilization from a discrete system of the form of a map $\xi_{i+1}=F\left(\xi_{i}, p\right)$, where $p \in \Re$ is an accessible parameter for control. This is equivalent to a parameter dependent map associated with a general surface, usually a Poincaré section. Let $\xi_{F}=F\left(\xi_{F}, p_{0}\right)$ denote the unstable fixed point on this section corresponding to an orbit in the chaotic attractor that one wants to stabilize. Basically, the control idea is to monitor the system dynamics until the neighborhood of this point is reached. After that, a proper small change in the parameter $p$ causes the next state $\xi_{i+1}$ to fall into the stable direction of the fixed point.

In order to overcome some limitations of the original OGY formulation such as control of orbits with large instability, measured by unstable eigenvalues, and orbits of high period, Hübinger et al. [21] introduced a semicontinuous control (SCC) method or local control method, which description is presented as follows.

The SCC method lies between the continuous and the discrete time control because one can introduce as many intermediate Poincaré sections, viewed as control stations, as it is necessary to achieve stabilization of a desirable UPO. Therefore, the SCC method is based on measuring transition maps of the system. These maps relate the state of the system in one Poincaré section to the next. In order to use $N$ control stations per forcing period $T$, one introduces $N$ equally spaced successive Poincaré sections $\Sigma_{n}, n=0, \ldots,(N-1)$. Let $\xi_{F}^{n} \in \Sigma_{n}$ be the intersections of the UPO with $\Sigma_{n}$ and $F^{(n, n+1)}$ be the mapping from one control station $\Sigma_{n}$ to the next one $\Sigma_{n+1}$. Hence, one considers the map

$$
\xi_{F}^{n+1}=F^{(n, n+1)}\left(\xi_{F}^{n}, p^{n}\right)
$$

A linear approximation of $F^{(n, n+1)}$ around $\xi_{F}^{n}$ and $p_{0}$ is considered as follows:

$$
\delta \xi^{n+1} \cong A^{n} \delta \xi^{n}+w^{n} \delta p^{n},
$$

where $\delta \xi^{n+1}=\xi^{n+1}-\xi_{F}^{n+1}, \delta p^{n}=p^{n}-p_{0}, A^{n}=D_{\xi^{n}} P^{(n, n+1)}\left(\xi_{F}^{n}, p_{0}\right)$, and $w^{n}=\frac{\partial P^{(n, n+1)}}{\partial p^{n}}\left(\xi_{F}^{n}, p_{0}\right)$.

Hübinger et al. [21] analyze the possibility of the eigenvalues of $A^{n}$ be complex numbers and then they use the fact that the linear mapping $A^{n}$ deforms a sphere around $\xi_{F}^{n}$ into an ellipsoid around $\xi_{F}^{n+1}$. Therefore, singular value decomposition (SVD),

$$
A^{n}=U^{n} W^{n}\left(V^{n}\right)^{T}=\left\{u_{u}^{n} u_{s}^{n}\right\}\left[\begin{array}{cc}
\sigma_{u}^{n} & 0 \\
0 & \sigma_{s}^{n}
\end{array}\right]\left\{\begin{array}{ll}
v_{u}^{n} & v_{s}^{n}
\end{array}\right\}^{T},
$$

is employed in order to determine the directions $v_{u}^{n}$ and $v_{s}^{n}$ in $\Sigma_{n}$ which are mapped onto the largest, $\sigma_{u}^{n} u_{u}^{n}$, and shortest, $\sigma_{s}^{n} u_{s}^{n}$, semi-axis of the ellipsoid in $\Sigma_{n+1}$, respectively. Here, $\sigma_{u}^{n}$ and $\sigma_{s}^{n}$ are the singular values of $A^{n}$.

Korte et al. [23] establish the control target as being the adjustment of $\delta p^{n}$ such that the direction $v_{s}^{n+1}$ on the map $n+1$ is obtained, resulting in a maximal shrinking on map $n+2$. Therefore, it demands $\delta \xi^{n+1}=\alpha v_{s}^{n+1}$, where $\alpha \in \Re$. Hence, from Eq. (2) one has that

$$
A^{n} \delta \xi^{n}+w^{n} \delta p^{n}=\alpha v_{s}^{n+1},
$$

which is a relation from what $\alpha$ and $\delta p^{n}$ can be conveniently chosen.

\subsection{State space reconstruction using delay coordinates}

The analysis of nonlinear dynamical systems from time series involves state space reconstruction, which the basic idea is that a signal contains information about unobserved state variables that can be used to predict the present state. Therefore, a scalar time series, $s(t)$, may be used to construct a vector time series that is topologically equivalent to the original dynamics. The state space reconstruction needs to form a coordinate system to capture the structure 
of orbits in state space which could be done using lagged variables, $s(t+\tau)$, where $\tau$ is the time delay. Then, considering an experimental signal, $s(n), n=1,2,3, \ldots, N$, where $t=t_{0}+(n-1) \Delta t$, it is possible to use a collection of time delays to create a vector in a $D_{e}$-dimensional space,

$$
u(t)=\left\{s(t), s(t+\tau), \ldots, s\left(t+\left(D_{e}-1\right) \tau\right)\right\}^{T}
$$

The method of delays has become popular for dynamical reconstruction, however, the choice of the delay parameters, $\tau$ - time delay, and $D_{e}$ - embedding dimension, may be a difficult task. Many researches have been developed considering the better approaches to estimate delay parameters for different kinds of time series.

As shown in [9], chaos control employing state space reconstruction by delay coordinates leads to a map $F^{(n, n+1)}$ that will depend on all parametric changes that influence the system in the time interval $t^{n}-\tau \leqslant t \leqslant t^{n}$, that is, $\delta p^{n}, \delta p^{n-1}, \ldots, \delta p^{n-r}$ with $r$ being the largest integer value such that $\delta p^{n-r}$ lies in this interval. Hence, the use of delay coordinates implies that the following map is considered, instead of the one shown in Eq. (1):

$$
\xi_{F}^{n+1}=F^{(n, n+1)}\left(\xi_{F}^{n}, \delta p^{n}, \delta p^{n-1}, \ldots, \delta p^{n-r}\right)
$$

Taking the linear approximation of $F^{(n, n+1)}$ around $\xi_{F}^{n}$ and $p_{0}$ using deviation variables $\delta \xi^{n+1}=\xi^{n+1}-\xi_{F}^{n+1}$ :

$$
\delta \xi^{n+1} \cong A^{n} \delta \xi^{n}+B_{0}^{n} \delta p^{n}+B_{1}^{n} \delta p^{n-1}+\ldots+B_{r}^{n} \delta p^{n-r},
$$

where, $A^{n}=D_{\xi^{n}} F^{(n, n+1)}\left(\xi_{F}^{n}, \delta p^{n}, \delta p^{n-1}, \ldots, \delta p^{n-r}\right)$ and $B_{i}^{n}=D_{\delta p^{n-i}} F^{(n, n+1)}\left(\xi_{F}^{n}, \delta p^{n}, \delta p^{n-1}, \ldots, \delta p^{n-r}\right)$.

SVD procedure is employed again and the resulting linear system is given by:

$$
A^{n} \delta \xi^{n}+B_{0}^{n} \delta p^{n}+B_{1}^{n} \delta p^{n-1}+\ldots+B_{r}^{n} \delta p^{n-r}=\alpha v_{s}^{n+1},
$$

from what $\alpha$ and $\delta p^{n}$ can be conveniently calculated. All the local dynamical properties of the control points can be extracted from state space reconstruction and one must wait until the system dynamics reaches the neighborhood of any of them to adequately perturb the control parameter.

\subsection{State space reconstruction using extended state observers}

A state observer may be understood as an auxiliary system that is employed to estimate a non-observable state of a system [22,25]. The observer is a very useful tool for receiving the information of the variables of a system that are otherwise unknown. For this reason, it is usually employed in control systems where it is necessary the full knowledge of the plant states from an incomplete observation. In general, the use of state observers is related to estimating, controlling and also detecting and identifying failures in dynamical systems.

This contribution uses the idea of the extended state observers (ESO) to promote state space reconstruction. In order to introduce the basic ideas of this procedure, consider a general nonlinear system given by:

$$
\left\{\begin{array}{l}
\dot{x}_{1}=x_{2} \\
\dot{x}_{2}=f\left(x_{1}, x_{2}, w(x, t)\right)+b_{0} u(x, t) \\
y=x_{1}
\end{array}\right.
$$

where $f$ is a nonlinear function that represents the dynamics of the system and the disturbance, $w(x, t)$ is the external unknown input disturbance, $u(x, t)$ is the control signal and $y$ is the observable output. Notice that $y=x_{1}$ is the only measured variable and the parameter $b_{0}$ is known. Therefore, it is necessary to estimate $x_{2}$ in order to determine the full state of the system.

Nonlinear systems have many types of uncertainties, such as imperfections of mechanisms, unknown nonlinearities and parameters, which make impossible to obtain an exact function $f$. Since classical state observer designs, including high-gain and sliding-mode observers, depend on the perfect knowledge of the system dynamics, Han [19] suggests an alternative method called extended state observer as an approach to deal with the estimation of states of dynamics that do not have a mathematical model. With this aim, the system Eq. (8) is augmented as

$$
\left\{\begin{array}{l}
\dot{x}=A x+B u+E h \\
y=C x
\end{array}\right.
$$

where 


$$
A=\left[\begin{array}{lll}
0 & 1 & 0 \\
0 & 0 & 1 \\
0 & 0 & 0
\end{array}\right], B=\left\{\begin{array}{l}
0 \\
b_{0} \\
0
\end{array}\right\}, C=\left\{\begin{array}{lll}
1 & 0 & 0
\end{array}\right\}, E=\left\{\begin{array}{l}
0 \\
0 \\
1
\end{array}\right\}
$$

Notice that, now, vector $x$ is defined by, $x=\left\{x_{1} x_{2} x_{3}\right\}^{T}$, where variable $x_{3}=f\left(x_{1}, x_{2}, w(x, t)\right)$ represents an extended state. It should be pointed out that, both $f$ and its derivative $h=\dot{f}$ are unknown. However, by making $f$ as a state, it is now possible to estimate it by using a state observer. Han [19] proposed the following form:

$$
\dot{\hat{x}}=A \hat{x}+B u+L G(e)
$$

Here, $\hat{x}=\left\{\hat{x}_{1} \hat{x}_{2} \hat{x}_{3}\right\}^{T}$ are estimated values of vector $x$. Notice that $\hat{x}_{3}$ represents an estimative of $f$, and $e=x_{1}-\hat{x}_{1}$ represents the error associated with the estimation. Moreover, $L$ is the observer gain vector, which can be obtained using known method such as pole placement method. In this equation, the following vectors are used:

$$
L=\left\{\beta_{1} \beta_{2} \beta_{3}\right\}^{T} \quad G(e)=\left\{g_{1}(e) g_{2}(e) g_{3}(e)\right\}
$$

The gain of the observer is chosen in order to obtain a good estimative for the system variables. Therefore, function $g_{i}$ is defined as a modified exponential gain function [45]:

$$
g_{i}\left(e, \alpha_{i}, \delta\right)=\left\{\begin{array}{l}
|e|^{\alpha_{i}} \operatorname{sign}(e), \quad|e|>\delta \\
\frac{e}{\delta^{1-\alpha_{i}}}, \quad|e| \leqslant \delta
\end{array}\right.
$$

The parameter $\delta$ is a small number used to limit the gain in the neighborhood of the origin. This procedure prevents excessive gain when error is small avoiding high frequency chattering. Therefore, when $0<\alpha_{i}<1, g_{i}$ yields high gain for small errors, $|e| \leqslant \delta$. On the other hand, when $\alpha_{i}<0$, it occurs a reduction of the observed error.

The definition of the gain is close related to the dynamics characteristics of the system. Usually, the first trial considers a linear gain $\left(\alpha_{i}=1, i=1,2,3\right), g_{i}\left(e, \alpha_{i}, \delta\right)=e(i=1,2,3)$. Under this assumption, the pole placement method can be used for the initial design of this observer. Nonlinearities can be added in order to improve the performance of the observer. Nevertheless, it is important to say that nonlinear gain functions introduce higher complexity in the estimation algorithm [15].

The stability of the observer is assured from an appropriate choice of parameters $\beta_{i}$. The stability of a linear extended state observer can be analyzed subtracting the observer Eq. (10) from system Eq. (9). This procedure gives an equation for the error dynamics:

$$
\dot{e}=A_{e} e+E h
$$

where

$$
A_{e}=A-L C=\left[\begin{array}{lll}
-\beta_{1} & 1 & 0 \\
-\beta_{2} & 0 & 1 \\
-\beta_{3} & 0 & 0
\end{array}\right]
$$

The stability of the error dynamics is associated with the roots of the characteristic polynomial of $A_{e}$, since $h$ is bounded:

$$
\lambda(s)=s^{3}+\beta_{1} s^{2}+\beta_{2} s+\beta_{3}=0
$$

In order to tune the linear ESO, the roots must be all in the open left-half plane. Gao [25] defines the $\omega_{o^{-}}$ parameterization where all observer eigenvalues must be equal to $-\omega_{o}$. Therefore, $L=\left\{\beta_{1} \beta_{2} \beta_{3}\right\}^{T}$ becomes a function of $\omega_{o}$, which is denoted as the bandwidth of the observer. Then,

$$
\lambda(s)=s^{3}+\beta_{1} s^{2}+\beta_{2} s+\beta_{3}=\left(s+\omega_{o}\right)^{3}=0
$$

Solving this equation, one obtains the following relationship between $\beta_{i}$ and $\omega_{o}: \beta_{1}=3 \omega_{0}, \beta_{2}=3 \omega_{o}^{2}, \beta_{3}=\omega_{o}^{3}$. 
Table 1

Experimental values of parameters

\begin{tabular}{ccccccc}
\hline$a(\mathrm{~m})$ & $b(\mathrm{~m})$ & $d(\mathrm{~m})$ & $D(\mathrm{~m})$ & $I\left(\mathrm{~kg} \mathrm{~m}^{4}\right)$ & $k(\mathrm{~N} / \mathrm{m})$ & $m(\mathrm{~kg})$ \\
\hline $1.6 \times 10^{-1}$ & $6.0 \times 10^{-2}$ & $2.9 \times 10^{-2}$ & $9.2 \times 10^{-2}$ & $1.876 \times 10^{-4}$ & 4.736 & $1.6 \times 10^{-2}$ \\
\hline
\end{tabular}

Table 2

Values of adjustable parameters

\begin{tabular}{ccc}
\hline $\bar{\omega}(\mathrm{rad} / \mathrm{s})$ & $\zeta\left(\mathrm{kg} . \mathrm{m}^{2} / \mathrm{s}\right)$ & $\Delta \mathrm{l}(\mathrm{m})$ \\
\hline 5.15 & $5.575 \times 10^{-5}$ & 0 \\
\hline
\end{tabular}

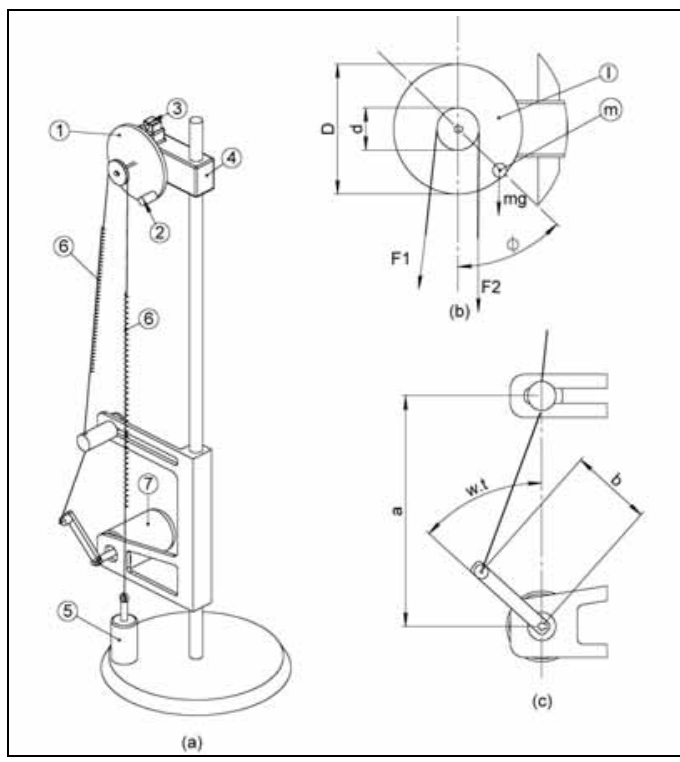

(d)

Fig. 1. Nonlinear pendulum. (a) Physical Model. (1) Metallic disc; (2) Lumped mass; (3) Magnetic damping device; (4) Rotary Motion Sensor; (5) Actuator; (6) String-spring device; (7) Electric motor. (b) Parameters and forces on the metallic disc. (c) Parameters from driving device. (d) Experimental apparatus.

\section{Nonlinear pendulum}

As a mechanical application of the procedures presented in this article, a nonlinear pendulum is considered. Nonlinear pendulum is one of the paradigms in the study of physics and natural phenomena, being employed in different situations to analyze bifurcations and chaos. Moreover, an additional motivation of the proposed pendulum is an experimental set up, previously analyzed in [13,32]. Pereira-Pinto et al. [30] present a mathematical model to describe the dynamical behavior of the pendulum. Here, it is shown just the equations of motion. For more details, see the cited reference.

The considered nonlinear pendulum is shown in Fig. 1. The right side presents the experimental apparatus while the left side shows a schematic picture. Basically, pendulum consists of an aluminum disc Eq. (1) with a lumped mass Eq. (2) that is connected to a rotary motion sensor Eq. (4). This assembly is driven by a string-spring device Eq. (6) that is attached to an electric motor Eq. (7) and also provides torsional stiffness to the system. A magnetic device Eq. (3) provides an adjustable dissipation of energy. An actuator Eq. (5) provides the necessary perturbations to stabilize this system by properly changing the string length.

The equations of motion of this pendulum are given by [30]: 


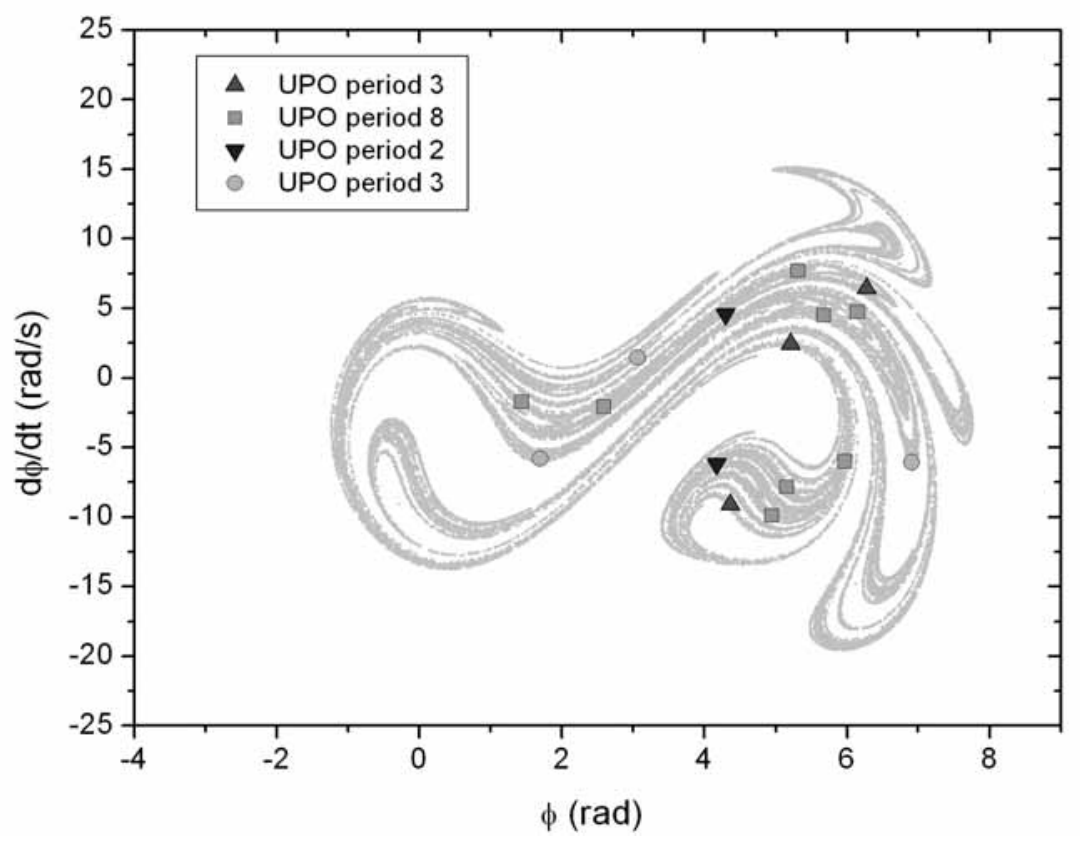

Fig. 2. Strange attractor in intermediate sections showing identified UPOs.
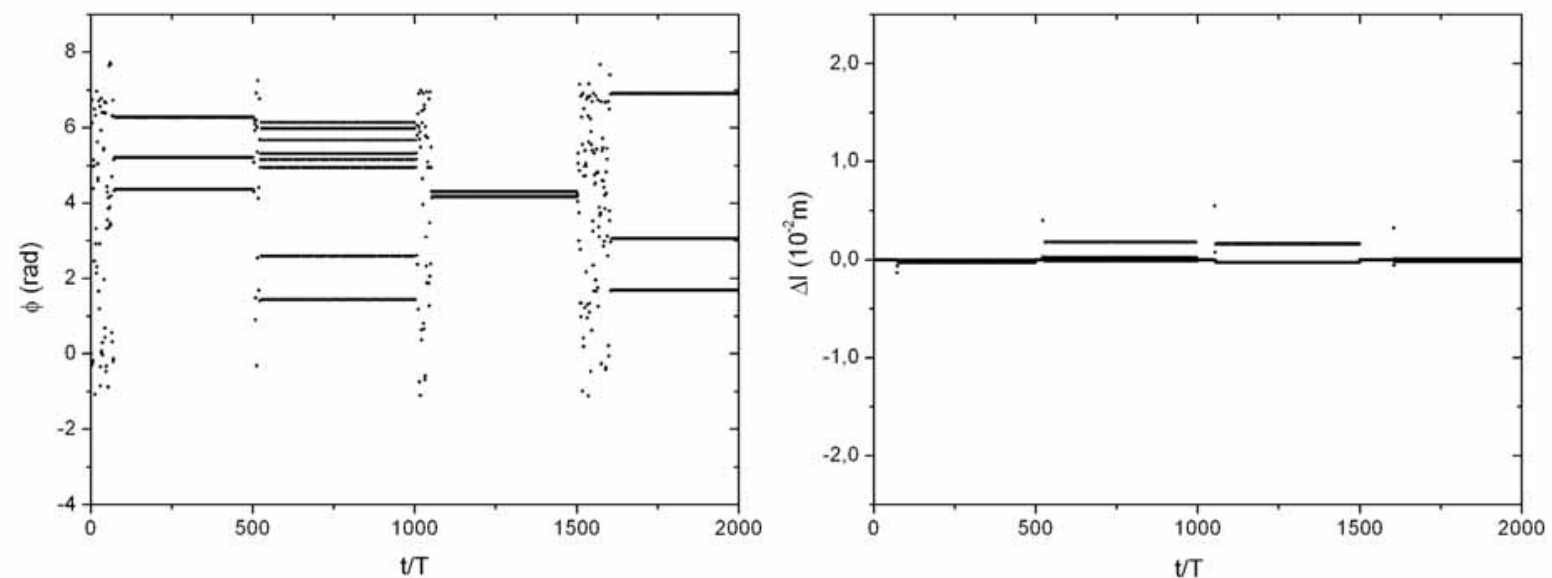

Fig. 3. Response under control. (a) Temporal alternating of UPOs in Poincaré section \#1. (b) Control signal

$$
\left\{\begin{array}{c}
\dot{\phi} \\
\ddot{\phi}
\end{array}\right\}=\left[\begin{array}{l}
0 \\
-\frac{k d^{2}}{2 I}-\frac{\zeta}{I}
\end{array}\right]\left\{\begin{array}{l}
\phi \\
\dot{\phi}
\end{array}\right\}+\left\{\begin{array}{l}
0 \\
\frac{k d}{2 I}\left(\sqrt{a^{2}+b^{2}-2 a b \cos (\bar{\omega} t)}-(a-b)-\Delta l\right)-\frac{m g D}{2 I} \sin (\phi)
\end{array}\right\}
$$

where $\bar{\omega}$ is the forcing frequency, $a$ defines the position of the guide of the string with respect to the motor, $b$ is the length of the excitation arm of the motor, $D$ is the diameter of the metallic disc and $d$ is the diameter of the driving pulley; $I$ is the total inertia of rotating parts, $m$ is the lumped mass and $\zeta$ is the dissipation parameter. The $\Delta l$ parameter is the length variation in the string provided by the linear actuator Eq. (5) shown in Fig. 1(a). This parameter is considered as the variation on the accessible parameter for control purposes.

The determination of parameters in equation of motion is done by considering the experimental setup of Franca and Savi [13]. Table 1 shows the parameters that are evaluated from the experimental setup. Moreover, values of 

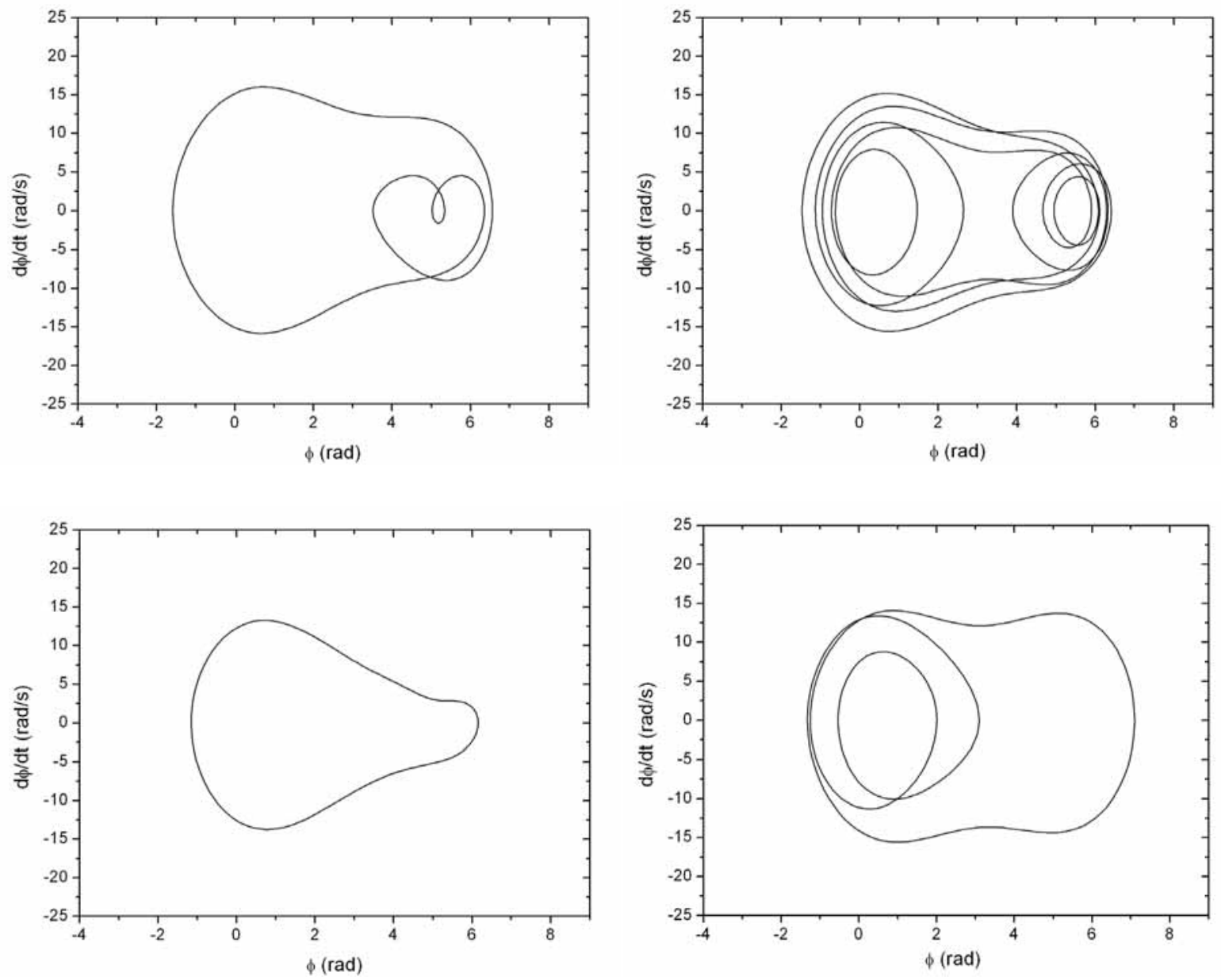

Fig. 4. Stabilized UPOs.

the adjustable parameters $\varpi$ and $\zeta$ are tuned to generate chaotic response in agreement to the experimental work. The $\Delta l$ parameter has a null value for the system without control action. Therefore, using the parameters presented in Table 2, it is possible to use a fourth-order Runge-Kutta scheme in order to perform numerical simulations of the equations of motion. These parameters are associated with chaos, assured evaluating Lyapunov exponents. By employing the algorithm proposed by Wolf et al. [46], one obtains the following spectrum that presents one positive value: $\lambda=\{+19.21,-5.19\}$.

\section{Controlling nonlinear pendulum}

The first stage of the control strategy is the identification of UPOs embedded in the chaotic attractor. The CR method [2] is employed with this aim. The value of the tolerance $r_{1}$ is chosen to be 0.003 and $r_{2}$ is set to be ten times $r_{1}$. Figure 2 presents a strange attractor of the motion showing points in the Poincar é section corresponding to some identified UPOs that will be stabilized in the next stage of control strategy. The SCC method is applied considering three control stations. Therefore, a total of four maps per forcing period are considered.

After the identification of the UPOs embedded in the Poincaré section \#1, the piercing of the same UPOs in the other three Poincaré sections is determined. Then, the local dynamics expressed by the Jacobian matrix and the sensitivity vector of the transition maps in a neighborhood of the fixed points are determined using the least-square 


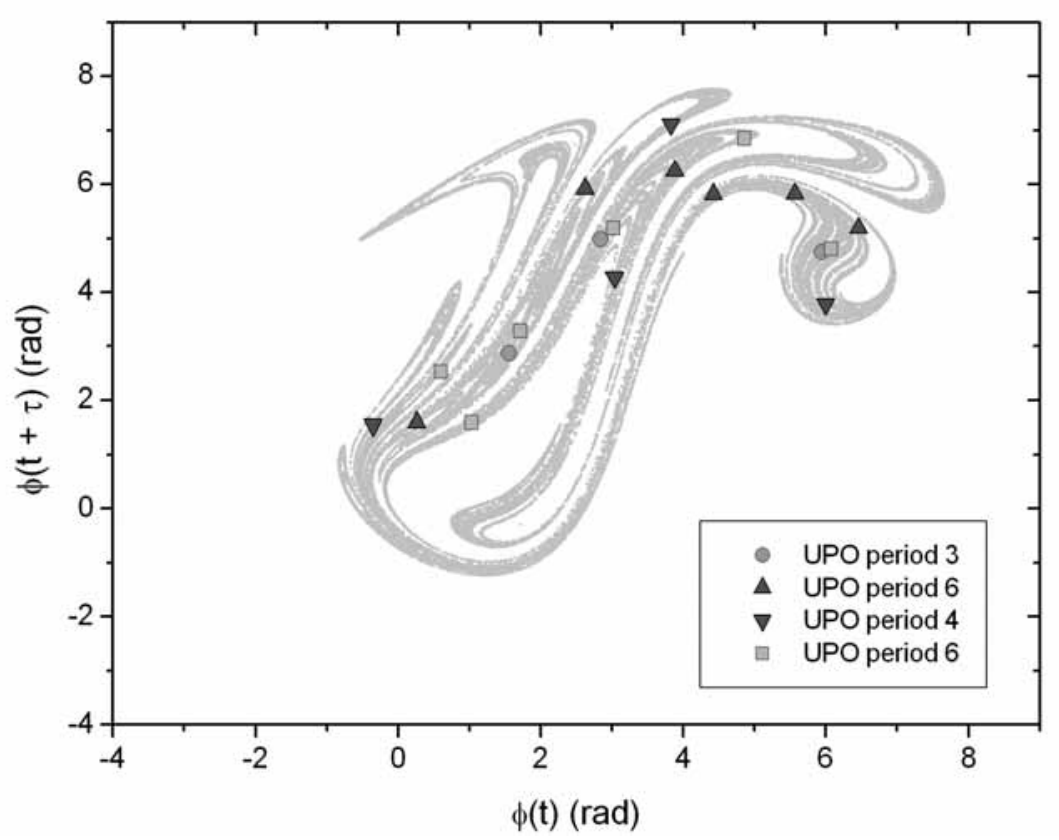

Fig. 5. Reconstructed strange attractor.
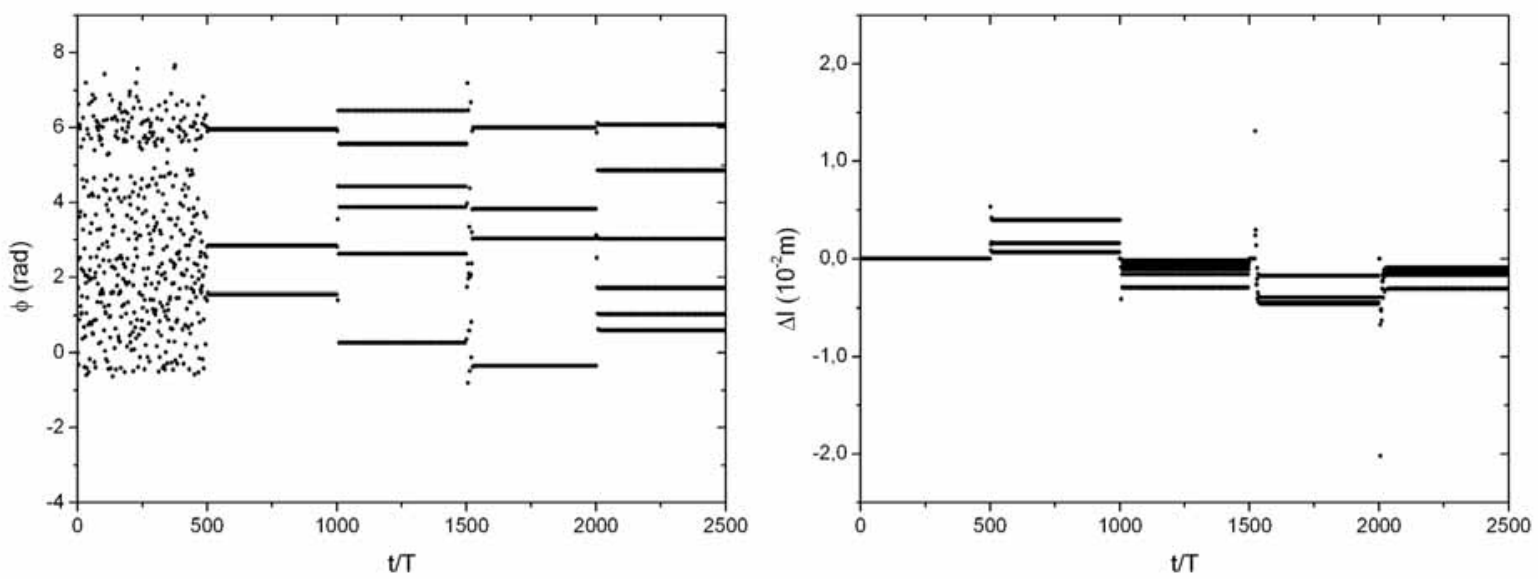

Fig. 6. Response under control. (a) Position of UPOs in Poincaré section \#1. (b) Control signal.

fit method [2,28]. After that, the SVD technique is employed for determining the stable and unstable directions near the next fixed point. The sensitivity vectors are evaluated allowing the trajectories to come close to a fixed point and then one perturbs the parameters by the maximum permissible value. In this case, a perturbation in $\Delta l_{\max }=20 \mathrm{~mm}$ is performed, fitting the resulting deviations $\left[\delta \xi^{n+1}(\Delta l)-A^{n} \delta \xi^{n}\right] / \Delta l$ from the next piercing by the least square procedure. After that, SCC method is employed to stabilize unstable periodic orbits and the parameter changes are calculated.

In order to exploit the possibilities of alternating the stabilized orbits with small changes in the control parameter, one performs a simulation that aims the stabilization of the following UPOs: a period-3 UPO in the first 500 forcing periods, a period-8 UPO between 500 and 1000 forcing periods, a period-2 UPO between 1000 and 1500 forcing periods and a period-3 UPO, different from the first one, between 1500 and 2000 forcing periods. Figure 3 shows 

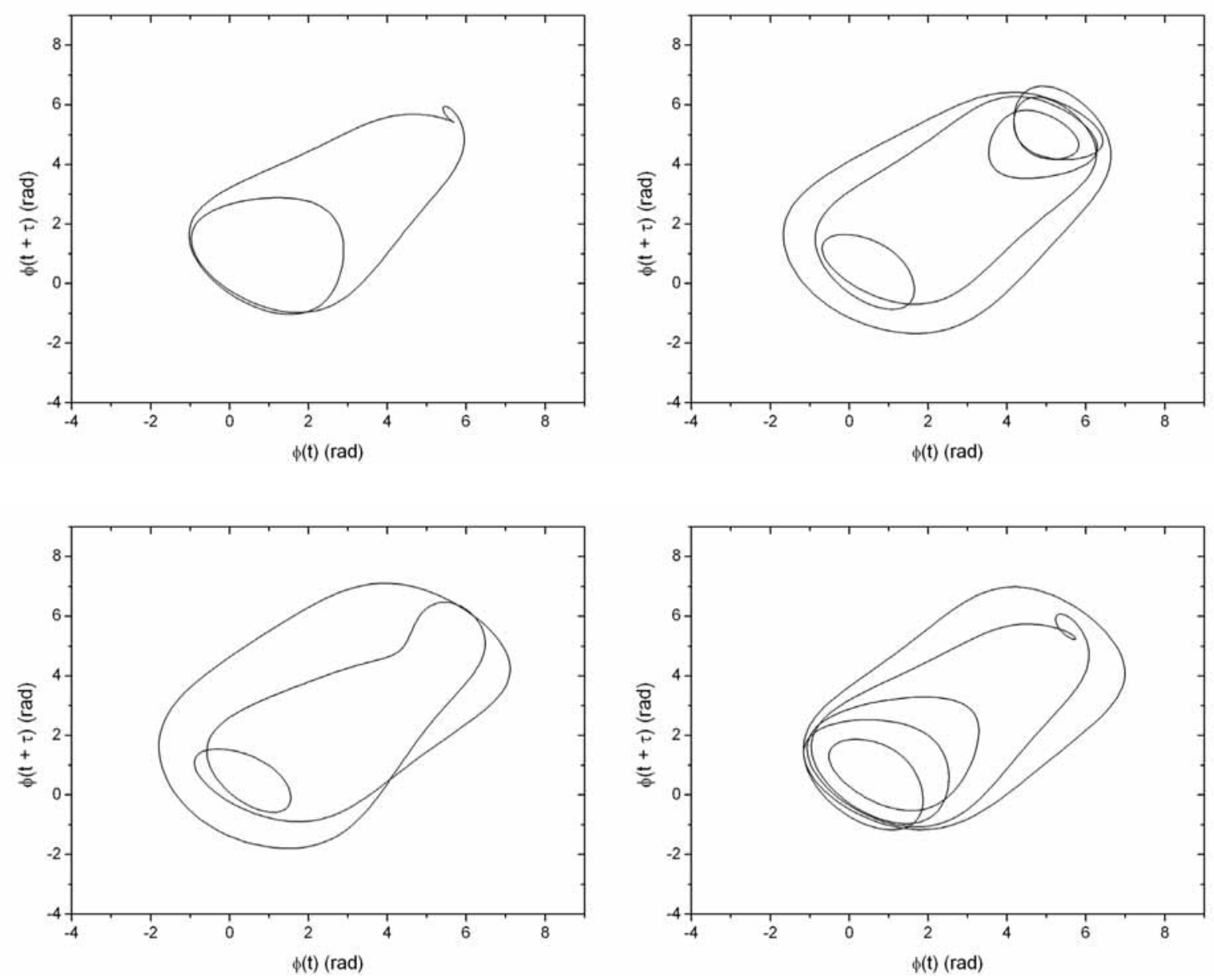

Fig. 7. Stabilized UPOs.

the system's dynamics in the Poincaré section \#1 during the actuation. Notice that different times are needed for the system to achieve the desired stabilization on a particular UPO. This happens because one must wait until the trajectory comes close enough to a control point to perform the necessary perturbation, exploiting the ergodicity property of the chaos. Moreover, it should be pointed out that, as expected, results show that unstable orbits are stabilized with small variations of control parameter after a transient, less than $2 \mathrm{~mm}$ in this case.

More details on the stabilized orbits due to SCC method are presented in Fig. 4, which shows different stabilized orbits. This example shows the flexibility of the systems represented by the change between two significantly different orbits, which is related to a quickly reaction of the system.

\section{Controlling using delay coordinates}

In this section, it is assumed that a scalar time series of angular position is acquired with sampling time, $\Delta \mathrm{t}$. Therefore, in order to reconstruct the dynamics of the system from time series, delay coordinates is employed. The average mutual information method is employed to determine time delay [14] while the false nearest neighbors method is used to estimate embedding dimension [35]. Figure 5 shows the reconstructed Poincar é section related to chaotic behavior. State space reconstruction is considered with time delay $\tau=24 \Delta t$ and a embedding dimension $D_{e}=2[13]$. 


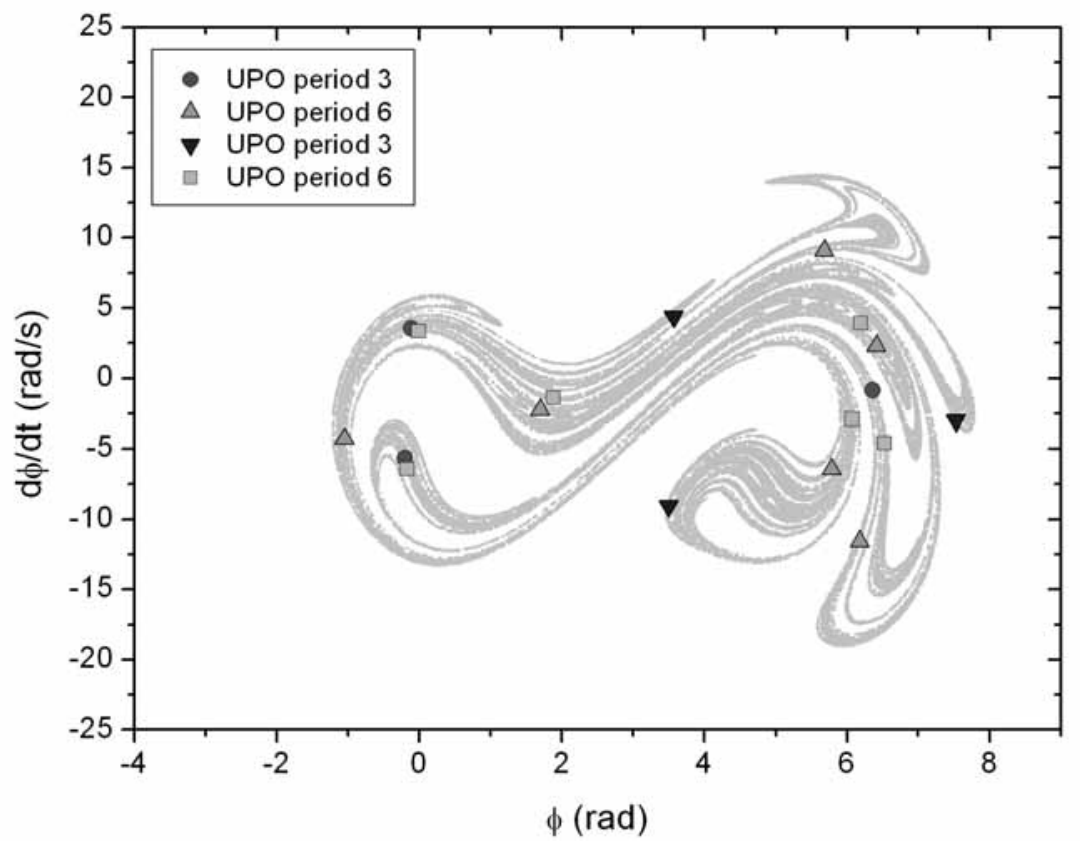

Fig. 8. Unstable periodic embedded in the reconstructed attractor.

After the identification of the UPOs embedded in the Poincaré section \#1 (see Fig. 5), the piercing of the same UPO in the other three Poincaré sections is determined. Then, the local dynamics expressed by the Jacobian matrix of the transition maps are determined using the least-square method [2]. The sensitivity vectors $B_{0}^{n}$ and $B_{1}^{n}$ are determined as proposed by Dressler \& Nitsche [9]. Subsequently, the SVD technique is employed to determine stable and unstable directions near the next control point and the necessary perturbation on $\Delta l$ parameter is done when the system's trajectory enters in a neighborhood of a control point.

A simulation of the stabilization of some UPOs is performed aiming the following sequence of UPOs: period-3, period-6, period-4 and period-6, different from the first one. Figure 6 shows the system's position and parameter perturbation in the Poincaré section \#1 during the control procedure. Notice that the control is turned on after the first 500 forcing periods. It is clear that the control procedure is also able to perform UPO stabilization using delay coordinates.

Figure 7 shows the cited stabilized orbits. Again, it should be pointed out the capacity to change from one orbit to another with small perturbations.

\section{Controlling using extended state observers}

At this point, extended state observers are employed in order to perform state space reconstruction. The state space reconstruction using extended state observers has some advantages in chaos control. The method of delay coordinates, for example, leads to a map $F^{(n, n+1)}$ that will depend on all parametric changes that influence the system in the time interval $t^{n}-\tau \leqslant t \leqslant t^{n}$, that is, $\delta p^{n}, \delta p^{n-1}, \ldots, \delta p^{n-r}$ with $r$ being the largest integer value such that $\delta p^{n-r}$ lies in this interval $[9,31]$. Therefore, the use of extended state observers avoids these calculations, allowing a direct application of the SCC method.

Figure 8 presents a strange attractor of the motion showing points in the Poincar é section corresponding to some identified UPOs that will be stabilized in the next stage of control strategy. As in the previous analysis, the SCC method is applied considering four maps per forcing period.

The stabilization of the UPOs now considers the following sequence: during the first 500 forcing periods control is off. After that is stabilized a period-3 UPO between 500 and 1000 forcing periods. A period-6 UPO is then 

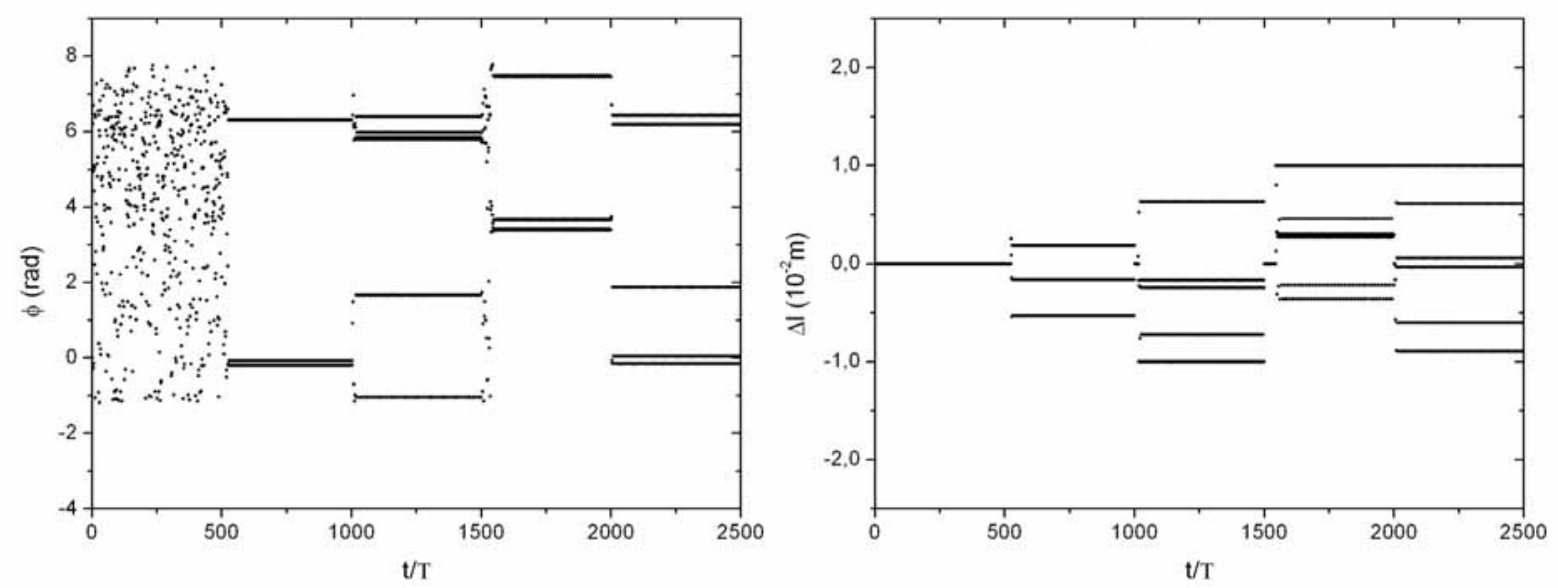

Fig. 9. Response under control. (a) Temporal alternating of UPOs in Poincaé section \#1. (b) Control signal

stabilized between 1000 and 1500 forcing periods. After that, is stabilized a period-3 UPO, different from the first one, between 1500 and 2000 forcing periods and finally a period-6, from 2000 and 2500 forcing periods. Figure 9 shows the system's dynamics in the Poincaré section \#1 during the actuation. Notice again that different times are needed for the system to achieve the desired stabilization on a particular UPO. Moreover, it should be pointed out that, as expected, results show that unstable orbits are stabilized with small variations of control parameter after a transient, less than $10 \mathrm{~mm}$ in this case.

Figure 10 shows the stabilized orbits, comparing with those obtained when all state variables are known. Notice that the state space reconstruction using extended state observer also estimates the variables values. In terms of control, again it is possible to observe the ability of the controller in stabilize a UPO.

\section{Conclusions}

This contribution discusses the control of chaos in a simulated nonlinear pendulum based on an experimental apparatus previously analyzed in [13,32]. In the first stage of the control process, the close-return method is employed to identify unstable periodic orbits (UPOs) embedded in the chaotic attractor. After that, the semi-continuous control (SCC) method is considered to stabilize desirable orbits. Least-square fit method is employed to estimate Jacobian matrixes and sensitivity vectors. Moreover, SVD decomposition is employed to estimate directions of unstable and stable manifolds in the vicinity of control points. Signals are generated by the numerical integration of the mathematical model. At first, it is considered that all variables are known. Simulations of control procedure show that SCC method is capable to perform stabilization of the nonlinear pendulum. After that, the analysis is done selecting a single variable as a time series and therefore, it is important to evaluate the effect of state space reconstruction on control. Delay coordinates and extended state observers are employed with this aim. These techniques are employed to stabilize some of the identified UPOs, confirming the possibility of using such approach to control chaotic behavior in mechanical systems using state space reconstruction. In general, it is worth mentioning that chaos control techniques are applicable to mechanical systems motivating further applications where great flexibility is desirable.

\section{Acknowledgement}

The authors acknowledge the support of the Brazilian Research Council CNPq. 

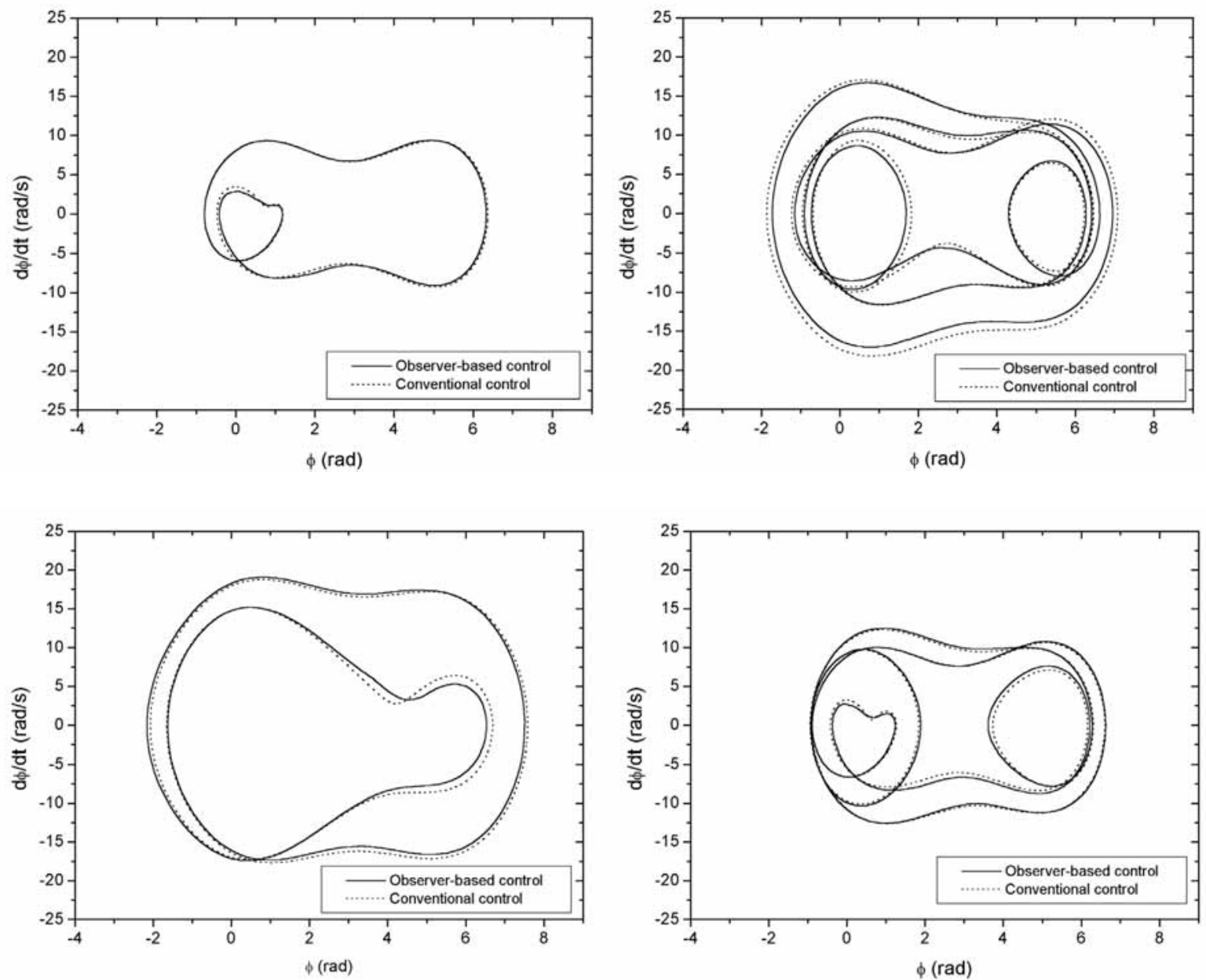

Fig. 10. Stabilized UPOs.

\section{References}

[1] B.R. Andrievskii and A.L. Fradkov, Control of chaos: methods and applications, II - Applications, Automation And Remote Control 65(4) (2004), 505-533.

[2] D. Auerbach, P. Cvitanovic, J.-P. Eckmann, G. Gunaratne and I. Procaccia, Exploring chaotic motion through periodic orbits, Physical Review Letters 58(23) (1987), 2387-2389.

[3] C.J. Begley and L.N. Virgin, On the OGY control of an impact-friction oscillator, Journal of Vibration and Control 7(6) (2001), 923-931.

[4] S. Boccaletti, C. Grebogi, Y.-C. Lai, H. Mancini and D. Maza, The control of chaos: theory and applications, Physics Reports (329) (2000), 103-197.

[5] P. Chanfreau and H. Lyyjynen, Viewing the efficiency of chaos control, Journal of Nonlinear Mathematical Physics 6(3) (1999), 314-331.

[6] G. Chen, Introduction to Chaos Control and Anti-control, In: Chapter 6, in: Advanced Topics in Nonlinear Control Systems, T.P. Leung and H.-S. Qin, eds, World Scientific Pub. Co., Singapore, 2001, pp. 193-245.

[7] W.L. Ditto, M.L. Spano and J.F. Lindner, Techniques for the Control of Chaos, Physica D 86 (1995), 198-211.

[8] W.L. Ditto and K. Showalter, Introduction: control and synchronization of chaos, Chaos 7(4) (1997), 509-511.

[9] U. Dressler and G. Nitsche, Controlling chaos using time delay coordinates, Physical Review Letters 68(1) (1992), 1-4.

[10] L.J. Dubé and P. Després, The Control of Dynamical Systems - Recovering Order from Chaos, in: The Physics of Electronic and Atomic Collisions, Y. Itikawa, ed., AIP Conference Proceedings 500, 2000, pp. 551-570.

[11] P. Faure and H. Korn, Is there chaos in the Brain? I. Concepts of Nonlinear Dynamics and Methods of Investigation, C.R. Acad. Sci. Paris, Life Sciences 324 (2003), 773-793.

[12] R. Ferriere and G.A. Fox, Chaos and evolution, Trends in Ecology and Evolution 10(12) (1995), 480-485.

[13] L.F.P. Franca and M.A. Savi, Distinguishing periodic and chaotic time series obtained from an experimental nonlinear pendulum, Nonlinear Dynamics 26 (2001), 253-271. 
[14] A.M. Fraser and H.L. Swinney, Independent coordinates for strange attractors from mutual information, Physical Review A 33 (1986), 1134-1140.

[15] Z. Gao, Scaling and Bandwidth-Parameterization Based Controller Tuning, In: American Control Conference, Denver, Colorado, 2003.

[16] L. Glass, Synchronization and rhythmic processes in physiology, Nature 410 (March 2001), 277-284.

[17] C. Grebogi and Y.-C. Lai, Controlling chaotic dynamical systems, Systems \& Control Letters 31 (1997), 307-312.

[18] G. Gunaratne, P.S. Linsay and M.J. Vinson, Chaos beyond onset: A comparison of theory and experiment, Physical Review Letters 63(1) (1989), 1-4.

[19] J. Han, A class of extended state observers for uncertain systems, Control and Decision 10 (1995), 85-88.

[20] H.-Y. Hu, Controlling Chaos of a Periodically Forced Nonsmooth Mechanical System, Acta Mechanica Sinica 11(3) (1995), $251-258$.

[21] B. Hübinger, R. Doerner, W. Martienssen, M. Herdering, R. Pitka and U. Dressler, Controlling Chaos Experimentally in Systems Exhibiting Large Effective Lyapunov Exponents, Physical Review E 50(2) (1994), 932-948.

[22] R.E. Kalman, A New Approach to Linear Filtering and Prediction Problems, Transactions of the ASME - Journal of Basic Engineering 82(Series D) (1960), 35-45.

[23] R.J. de Korte, J.C. Schouten and C.M.V. van den Bleek, Experimental Control of a Chaotic Pendulum with Unknown Dynamics Using Delay Coordinates, Physical Review E 52(4) (1995), 3358-3365.

[24] E.J. Kostelich and T. Schreiber, Noise Reduction in Chaotic Time-Series Data: A Survey of Common Methods, Physical Review E 48(3) (1993), 1752-1763.

[25] D.G. Luenberger, Observing the State of a Linear System, IEEE Transactions on Military Electronics MIL8 (1964).

[26] E.E.N. Macau, Exploiting Unstable Periodic Orbits of a Chaotic Invariant Set for Spacecraft Control, Celestial Mechanics \& Dynamical Astronomy 87(3) (2003), 291-305.

[27] F.C. Moon, A.J. Reddy and W.T. Holmes, Experiments in Control and Anti-control of Chaos in a Dry Friction Oscillator, Journal of Vibration and Control 9(3-4) (2003), 387-397.

[28] M. Otani and A.J. Jones, Guiding Chaotic Orbits, 130, Research Report, Imperial College of Science Technology and Medicine, London, 1997.

[29] E. Ott, C. Grebogi and J.A. Yorke, Controlling Chaos, Physical Review Letters 64(11) (1990), 1196-1199.

[30] F.H.I. Pereira-Pinto, A.M. Ferreira and M.A. Savi, Chaos Control in a Nonlinear Pendulum Using a Semi-Continuous Method, Chaos, Solitons and Fractals 22(3) (2004), 653-668.

[31] F.H.I. Pereira-Pinto, A.M. Ferreira and M.A. Savi, State Space Reconstruction Using Extended State Observers to Control Chaos in a Nonlinear Pendulum, International Journal of Bifurcation and Chaos 15(12) (2005), 4051-4063.

[32] E.G.F. Pinto and M.A. Savi, Nonlinear Prediction of Time Series Obtained from an Experimental Pendulum, Current Topics in Acoustical Research - Research Trends 3 (2003), 151-162.

[33] K. Pyragas, Continuous Control of Chaos by Self-controlling Feedback, Physics Letters A 170 (1992), 421-428.

[34] T. Ritz, A.S.Z. Schweinsberg, U. Dressler, R. Doerner, B. Hübinger and W. Martienssen, Chaos Control with Adjustable Control Times, Chaos, Solitons \& Fractals 8(9) (1997), 1559-1576.

[35] C. Rhodes and M. Morari, False-nearest-neighbors Algorithm and Noise-corrupted Time Series, Physical Review E 55(5) (1997), 61626170 .

[36] P. Schmelcher and F.K. Diakonos, General Approach to the Localization of Unstable Periodic Orbits in Chaotic Dynamical Systems, Physical Review E 57(3) (1998), 2739-2746.

[37] T. Shinbrot, C. Grebogi, E. Ott and J.A. Yorke, Using Small Perturbations to Control Chaos, Nature 363 (1993), $411-417$.

[38] P. So and E. Ott, Controlling Chaos Using Time Delay Coordinates via Stabilization of Periodic Orbits, Physical Review E 51(4) (1995), 2955-2962.

[39] M.L. Spano, W.L. Ditto and S.N. Rauseo, Exploitation of Chaos for Active Control: An Experiment, Journal of Intelligent Material Systems and Structures 2(4) (1990), 482-493.

[40] F. Takens, Detecting Strange Attractors in Turbulence, in: Proceedings of the Symposion on Dynamical Systems and Turbulence, University of Warwick, 1979-1980, D.A. Rand and L.S. Young, eds, Springer, Berlin, 1981.

[41] K. Yagasaki and T. Uozumi, Controlling Chaos in a Pendulum Subjected to Feedforward and Feedback Control, International Journal of Bifurcation and Chaos 7(12) (1997), 2827-2835.

[42] K. Yagasaki and S. Yamashita, Controlling Chaos Using Nonlinear Approximations for a Pendulum with Feedforward and Feedback Control, International Journal of Bifurcation and Chaos 9(1) (1999), 233-241.

[43] E.A. Wan and R. van der Merwe, in: The Unscented Kalman Filter, S. Haykin, ed., Kalman Filtering and Neural Networks, Wiley Publ, 2001.

[44] R.Q. Wang and Z.J. Jing, Chaos Control of Chaotic Pendulum System, Chaos, Solitons \& Fractals 21(1) (2004), $201-207$.

[45] W. Wang and Z. Gao, A Comparison Study of Advanced State Observer Design Techniques, In: American Control Conference, Denver, Colorado, June, 2003.

[46] A. Wolf, J.B. Swift, H.L. Swinney and J.A. Vastano, Determining Lyapunov Exponents from a Time Series, Physica D 16 (1985), $285-317$. 

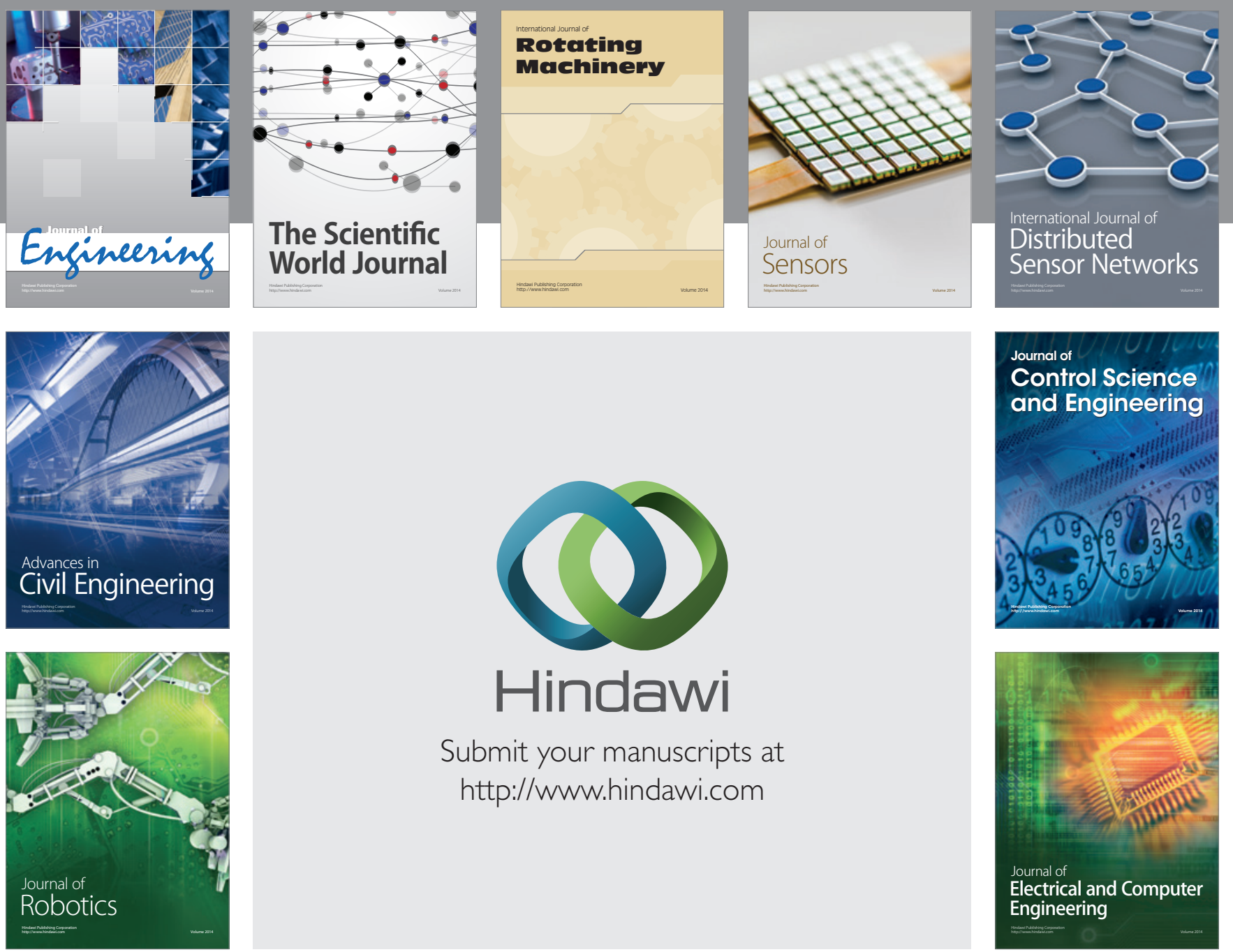

Submit your manuscripts at

http://www.hindawi.com
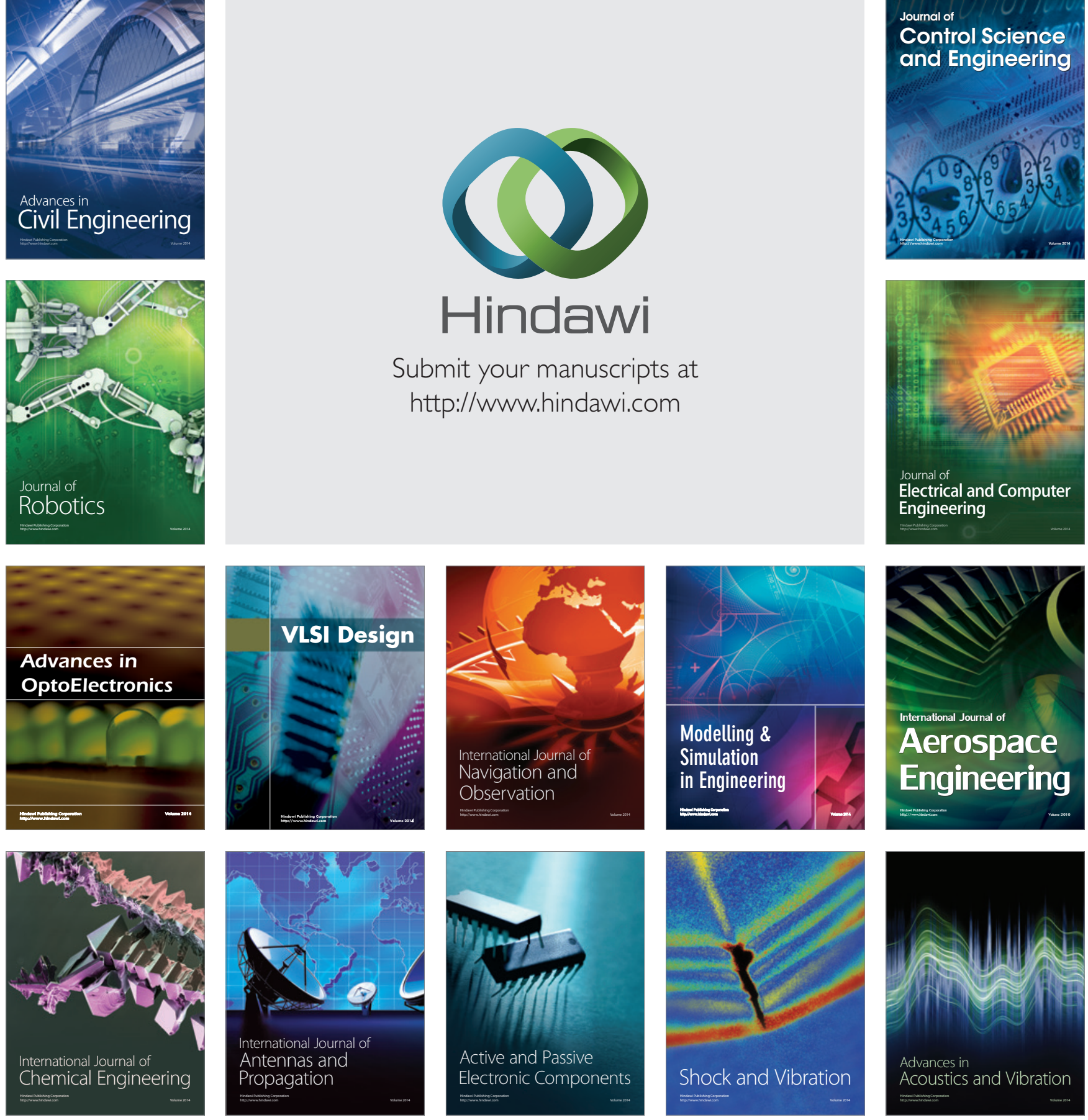